\title{
Simplicial Homology for Future Cellular Networks
}

\author{
Anaïs Vergne, Laurent Decreusefond, Philippe Martins, Senior Member, IEEE
}

\begin{abstract}
Simplicial homology is a tool that provides a mathematical way to compute the connectivity and the coverage of a cellular network without any node location information. In this article, we use simplicial homology in order to not only compute the topology of a cellular network, but also to discover the clusters of nodes still with no location information. We propose three algorithms for the management of future cellular networks. The first one is a frequency auto-planning algorithm for the selfconfiguration of future cellular networks. It aims at minimizing the number of planned frequencies while maximizing the usage of each one. Then, our energy conservation algorithm falls into the self-optimization feature of future cellular networks. It optimizes the energy consumption of the cellular network during off-peak hours while taking into account both coverage and user traffic. Finally, we present and discuss the performance of a disaster recovery algorithm using determinantal point processes to patch coverage holes.
\end{abstract}

Index Terms-Future cellular networks, Self-Organizing Networks, simplicial homology.

\section{INTRODUCTION}

$\mathbf{L}$ ONG Term Evolution (LTE) is the 3GPP standard specified in Releases 8 and 9. Its main goal is to increase both capacity and speed in cellular networks. Indeed, cellular network usage has changed over the years and bandwidth hungry applications, as video calls, are now common. Achieving this goal for both capacity and speed costs a lot of money to the network operator. A solution to limit operation expenditures is the introduction of Self-Organizing Networks (SON) in LTE systems. 3GPP standards have indeed identified self-organization as a necessity for future cellular networks [1]. Self-organization is the ability for a cellular network to automatically configure itself and adapt its behavior without any manual intervention. Therefore, SON features can be divided into self-configuration, self-optimization, and selfhealing functions. We will define and describe the features we are interested in, for a further reading a full description of SON in LTE can for instance be found in [2].

First, self-configuration functions aim at the plug-and-play paradigm: new transmitting nodes should be automatically configured and integrated to the existing network. Upon arrival of a new node, the neighboring nodes update their dynamic neighbor tables thanks to the Automatic Neighbor Relation (ANR) feature. Among self-configuration functions, we can find the dynamic frequency auto-planning. It is a known problem from spectrum-sensing cognitive radio where equipments are designed to use the best wireless channels in order to limit interference [3]. The different nodes of the secondary

Manuscript created on September 11, 2014.

A. Vergne is with the Geometrica team, Inria Saclay - Ile de France, Palaiseau, France.

L. Decreusefond, and P. Martins are with the Network and Computer Science Department, Telecom ParisTech, Paris, France. cognitive network have to choose the best frequency to use in order to maximize the coverage and minimize the interference with the base stations of the primary network. A similar approach has been made in [4] for the spectrum allocation of femtocells. However, there is no strict hierarchy between the nodes of future cellular networks, all nodes pertain to the primary network. Therefore these solutions can not always be used here. Moreover, while in earlier releases, static frequency planning was preferred, it has become a critical point to allow dynamic configuration since the network has a dynamic behavior with arrivals and departures of base stations, and does not always follow a regular pattern with the introduction of femtocells and relays in heterogeneous networks.

The second main SON feature is the category of the selfoptimization functions, which defines the ability of the network to adapt its behavior to different traffic scenarios. Indeed, in LTE cellular networks, eNode-Bs (eNBs) have multiple configurable parameters. An example is output power, so cells sizes can be configured when capacity is the limitation rather than coverage. Moreover, fast and reliable X2 communication interfaces connect eNBs. So the whole network has the capability to adapt to different traffic situations. Then, users traffic can be observed via eNBs and User Equipments (UEs) measurements. Therefore, the self-optimization functions aim at using these traffic observations to adapt the whole network, and not only each cell independently, to the traffic situation. One case where self-optimization is often needed is the adaptation to off-peak hours. Typically a cellular network is deployed to match daily peak hours traffic requirements. Therefore during off-peak hours, the network is daily under-used. This leads to a huge unneeded amount of energy consumption. An idea is thus to switch-off some of the eNBs during off-peak hours, while other eNBs adjust their configuration parameters to keep the entire area covered. If the traffic grows, switchedoff eNBs can be woken up to satisfy the user demand.

The third and last of the SON main functions is self-healing. In future cellular networks, nodes would be able to appear and disappear at any time. Since the cellular network is not only constituted of operated base stations anymore, the operator does not control the arrivals or departures of nodes. But the disappearances of nodes can be more generalized: for example in case of a natural disaster (floods, earthquakes or tsunamis...), several nodes do disappear at once. The self-healing functions aim at reducing the impacts from the failures of nodes must it be in isolated cases, like the turning off of a Femtocells, or more serious cases where the whole network is damaged. We are interested in this latter case, where some of the nodes are completely destroyed. However cellular networks are not necessarily built with redundancy and then can be sensitive to such damages. Coverage holes can appear resulting in no signal for communication at all in a whole area. Paradoxically, 
reliable and efficient communication is especially needed in such situations. Therefore, solutions for damage recovery for the coverage of cellular networks are much needed.

In this article, we use simplicial homology to comply with the self-organization requirements of future cellular networks. Simplicial homology provides a way to represent any wireless network without any location information, and compute its topology. A cellular network is then represented by a combinatorial object called abstract simplicial complex, and its topology is characterized in two dimensions by the so-called first two Betti numbers: the number of connected components and the number of coverage holes. But the simplicial complex representation does not only allow the topology computation, but it also gives geographical information, such as which nodes are in some clusters, or which ones are more homogeneously distributed. We use this simplicial complex representation in three algorithms that answer three specific aspects of SON in future cellular networks.

First, we propose a frequency auto-planning algorithm which, for any given cellular network, provides a frequency planning minimizing the number of frequencies needed for a given accepted threshold of interference. The algorithm calls several instances of a reduction algorithm, introduced in [5], for the allocation of each frequency. Using simplicial complex representation combined to the reduction algorithm allows us to obtain a homogeneous coverage between frequencies. In a second part, we enhance the reduction algorithm to satisfy any user traffic. The reduction algorithm, as it is presented in [5], only satisfies perfect connectivity and coverage. However, in cellular networks, especially in urban areas, coverage is not the limiting factor, capacity is. So the optimal solution is not optimal coverage anymore but depends on the required traffic. We present an enhanced reduction algorithm to reach an optimally used network. Finally, we present an algorithm for disaster recovery of wireless networks first introduced in [6]. Given a damaged cellular network, the algorithm first adds too many nodes then runs the reduction algorithm of [5] to reach an optimal result. For the addition of new nodes we propose the use of a determinantal point process which has the inherent ability to locate areas with low density of nodes: namely coverage holes.

We thoroughly evaluate the performance of our three homology-based greedy algorithms. We provide complexity results and performance comparison with three graph-based greedy algorithms. We aim at comparing our homology approach to the graph approach to see the benefit of the use of homology. Since we propose three greedy homologybased algorithms, then the comparison with three graph-based algorithms is expected.

The remainder of this article is organized as follows. After a section on related work on self-configuration, self-optimization and recovery in future cellular networks done in Section II, we introduce simplicial homology as well as the reduction algorithm we use all along the article in Section III. Then in Section IV, we introduce our frequency auto-planning algorithm. The energy conservation algorithm is presented in Section V. We provide the disaster recovery algorithm description in Section VI. Finally, we conclude in Section VII.

\section{RELATED WORK}

\section{A. Self-configuration in future cellular networks}

During the deployment of a cellular network, its different nodes (eNBs, relays, femtocells) has to be configured. This configuration happens first at the deployment, then upon every arrival and departure of any node. The classic manual configuration done for previous generations of cellular networks can not be operated in future cellular networks: changes in the network occur too often. Moreover, the dissemination of private femtocells leads to the presence in the network of nodes with no access for manual support. So the future cellular networks are heterogeneous networks with no regular pattern for their nodes. They need to be able to self-configure themselves. The initial parameters that a node needs to configure are its IP adress, its neighbor list and its radio access parameters. IP adresses are out of the scope of this work, but we will discuss the two other parameters. The selection of the nodes to put on one's neighbor list can be based on the geographical coordinates of the nodes and take into account the antenna pattern and transmission power [7]. However, this approach does not consider changing radio environment, and requires exact location information which can be easy to obtain for eNBs, but not for Femtocells. The authors of [8] propose a better criterion for the configuration of the neighbor list: each node scans in real time the Signal to Interference plus Noise Ratio (SINR) from other nodes, then the nodes which SINR are higher than a given threshold are included in the neighbor list. The neighbor list of a node is then equivalent to connectivity information between nodes. This is the only information needed in order to build the simplicial complex representing a given cellular network.

Among radio access parameters, we can find frequency but also propagation parameters since the apparition of beamforming techniques via MIMO. Let us focus on the former which is the subject of Section IV. The frequency planning problem was first introduced for GSM networks. However the constraints were not the same: the frequency planning was static with periodic manual optimizations, and in simulations, base stations were regularly deployed along an hexagonal pattern. With the deployments of Femtocells, outdoor relays, and Picocells, future cellular networks vary from GSM network in two major points. First, cells do not follow a regular pattern anymore, then they can appear and disappear at any time. Therefore the frequency planning problem has to be rethought in an automatic way. A naive idea for frequency auto-planning would simply be applying the greedy coloring algorithm to the sparse interference graph [9]. However, even if the provided solution may be optimal for the number of needed frequencies, the utilization of each frequency can be disparate: one can be planned for a large number of nodes compared to another planned for only few of them. Then if the level of interference increase (more users, or more powered antennas), this could lead to communication problems for the over-used frequency, and a whole new planning is needed. On the contrary, a more homogeneous resource utilization can be more robust if interference increase, since there are less nodes using the same frequency on average. We provide 
here a frequency auto-planning algorithm which aims at a more homogeneous utilization of the resources. Moreover, the planning of frequency channels for new nodes that do not interfere with existing nodes while still providing enough bandwidth is still an open problem. It has been addressed in the cognitive radio field, but these algorithms usually enable opportunistic spectrum access [10]. However, it is not possible to extend this type of algorithm to the frequency allocation of new nodes in cellular networks. Indeed, the new nodes would be part of the primary network, with a quality of service to achieve, so their frequency allocation needs to be guaranteed and not opportunistic. In [4], the authors propose a spectrum allocation for femtocells in a cellular network that is more suited to our needs. However, the frequency planning of the femtocells occurs after the frequency planning of the main cells (eNBs). We propose an algorithm that do not distinguish between different types of cells. Indeed there are more types of cells than exactly two, relays fall in between and femtocells are not necessarily alike. In our algorithm, the planning of all the nodes: eNBs, relays or femtocells, is done together.

\section{B. Self-optimization in future cellular networks}

In order to ensure that future cellular networks are still efficient in terms of both Quality of Service (QoS) and costs, the self-configuration is not sufficient. Indeed, future cellular networks have the ability to adjust their parameters to match different traffic situation. Periodic optimization based on $\log$ reports, and operated centrally is not an effective solution in terms of speed and costs. That is why we need self-optimization. Self-optimization can be classified in three types depending on its goal. First we can consider load balancing optimization. There is multiple ways to adapt a cellular network to different loads: it is for example possible to adapt the resources available in different nodes. These schemes were mainly introduced for GSM [11], and then CDMA [12], but the universal frequency reuse of LTE and LTE-Advanced diminishes their applicability. Then one can adapt the traffic strategy with admission controls on given cells and forced handovers [13]. However, as the previous solution, it is not very suitable for OFDMA networks which require hard handover. Finally it is possible to modify the coverage of a node by changing either its antennas radiation pattern [14] or the output power [15]. We use this latter approach to reach an optimal result: we adapt the coverage radius of each node to be the minimum required to cover a given area.

The second type self-optimization is the capacity and coverage adaptation via the use of relay nodes [16], while the third is interference optimization. Our energy conservation algorithm presented in Section $\mathrm{V}$ could lie in this third category as the simplest approach towards interference control is switching off idle nodes. It is done based on cell traffic for Femtocells in [17]: after a given period of time in idle mode, the node puts itself on stand-by. However, if one wants to take into account the whole network, it has to consider the coverage of the network before disconnecting, which is not the case of Femtocells, which are by definition redundant to the base stations network. Without considerations of traffic, we proposed in [5] an algorithm that reduces power consumption in wireless networks by putting on stand-by some of the nodes without impacting the coverage. We can also cite [18] that proposes a game-theoretic approach in which nodes are put on stand-by according to a coverage function, but unmodified coverage is not guaranteed. In both these works, only coverage is taken into account. This approach could eventually fit the requirements of cellular network in non-urban cells, if their deployment has coverage redundancy. But it is not valid for urban cells, where it is not coverage but capacity that delimits cells. Our present algorithm goes a little bit further by adapting the switching-off of the nodes to the whole network situation, considering both traffic and coverage.

\section{Recovery in future cellular networks}

The first step of recovery in cellular networks is the detection of failures. The detection of the failure of a cell occurs when its performance is considerably and abnormally reduced. In [19], the authors distinguish three stages of cell outage: degraded, crippled and catatonic. This last stage matches with the event of a disaster when there is complete outage of the damaged cells. After detection, compensation from other nodes can occur through relay assisted handover for ongoing calls, adjustments of neighboring cell sizes via power compensation or antenna tilt. In [20], the authors not only propose a cell outage management description but also describe compensation schemes. These steps of monitoring and detection, then compensation of nodes failures are comprised under the self-healing functions of future cellular networks described in [21].

In Section VI, we are interested in what happens when selfhealing is not sufficient. In case of serious disasters, the compensation from remaining nodes and traffic rerouting might not be sufficient to provide service everywhere. In this case, the cellular network needs a manual intervention: the adding of new nodes to compensate the failures of former nodes. However a traditional restoration with brick-and-mortar base stations could take a long time, when efficient communication is particularly needed. In these cases, a recovery trailer fleet of base stations can be deployed by operators [22], it has been for example used by AT\&T after 9/11 events. But a question remains: where to place the trailers carrying the recovery base stations. An ideal location would be adjacent to the failed node. However, these locations are not always available because of the disaster, plus the recovery base stations may not have the same coverage radii than the former ones. Therefore a new deployment for the recovery base stations has to be decided, in which one of the main goal is complete coverage of damaged area. This can be viewed as a mathematical set cover problem, where we define the universe as the area to be covered and the subsets as the balls of radii the coverage radii. Then the question is to find the optimal set of subsets that cover the universe, considering there are already balls centered on the existing nodes. It can be solved by a greedy algorithm [23], $\epsilon$-nets [24], or furthest point sampling [25], [26]. But these mathematical solutions provide an optimal mathematical result that do not consider any flexibility at all in the choosing 
of the new nodes positions, and that can be really sensitive to imprecisions in the nodes positions.

For a further reading, a complete survey on SON for future cellular networks is given in [27].

\section{PRELIMINARIES}

\section{A. Simplicial homology}

First we need to remind some definitions from simplicial homology for a better understanding of the simplicial complex representation of cellular networks.

When representing a cellular network with only connectivity information (i.e. neighbors lists) available, one's first idea will be a neighbor graph, where nodes are represented by vertices, and an edge is drawn whenever two nodes are on each other neighbors list. However, the graph representation has some limitations; first of all there is no notion of coverage. Graphs can be generalized to more generic combinatorial objects known as simplicial complexes. While graphs model binary relations, simplicial complexes represent higher order relations. A simplicial complex is a combinatorial object made up of vertices, edges, triangles, tetrahedra, and their $n$ dimensional counterparts. Given a set of vertices $V$ and an integer $k$, a $k$-simplex is an unordered subset of $k+1$ vertices $\left[v_{0}, v_{1} \ldots, v_{k}\right]$ where $v_{i} \in V$ and $v_{i} \neq v_{j}$ for all $i \neq j$. Thus, a 0 -simplex is a vertex, a 1 -simplex an edge, a 2 -simplex a triangle, a 3-simplex a tetrahedron, etc.

Any subset of vertices included in the set of the $k+1$ vertices of a $k$-simplex is a face of this $k$-simplex. Thus, a $k$-simplex has exactly $k+1(k-1)$-faces, which are $(k-1)$-simplices. For example, a tetrahedron has four 3 -faces which are triangles. A simplicial complex is a collection of simplices which is closed with respect to the inclusion of faces, i.e. all faces of a simplex are in the set of simplices, and whenever two simplices intersect, they do so on a common face. An abstract simplicial complex is a purely combinatorial description of the geometric simplicial complex and therefore does not need the property of intersection of faces. For details about algebraic topology, we refer to [28].

Given an abstract simplicial complex, its topology can be computed via linear algebra computations. The so-called Betti numbers are defined to be the dimensions of the homology groups and are easily obtained by the rank-nullity theorem, of which a proof is given in [28]. But the Betti numbers also have a geometrical meaning. Indeed, the $k$-th Betti number of an abstract simplicial complex $X$ is the number of $k$ th dimensional holes in $X$. In two dimensions we are only interested in the first two Betti numbers: $\beta_{0}$ counts the number of 0-dimensional holes, that is the number of connected components, and $\beta_{1}$ counts the number of holes in the plane, i.e. coverage holes. Therefore computing the Betti numbers of an abstract simplicial complex representing a cellular network gives the topology of the initial network. For the remainder of the paper we may drop the adjective "abstract" from abstract simplicial complex, since every simplicial complex in this paper is abstract.

\section{B. Reduction algorithm}

In this section, we recall the steps of the reduction algorithm for abstract simplicial complexes presented in [5] that we will use all along this article. The algorithm takes as input an abstract simplicial complex: here it is the complex representing the cellular network, and a list of boundary vertices that can be given by the network operator. This list of boundary vertices is needed in order to define the area of the network and not shrink it during the process of reduction. Then the goal of the reduction algorithm is to remove vertices form the abstract simplicial complex without modifying its Betti numbers. That translates to a network by turning off nodes from the network without modifying nor its connectivity neither its coverage.

To cover an area, only 2 -simplices are needed. So the first step of the reduction algorithm is to characterize the superfluous 2-simplices of the complex for its coverage. To do that, we define a degree for every 2 -simplex:

Definition 1: We define the degree of a 2-simplex $\left[v_{0}, v_{1}, v_{2}\right]$ to be the size of the largest simplex it is part of:

$$
D\left[v_{0}, v_{1}, v_{2}\right]=\max \left\{d \mid\left[v_{0}, v_{1}, v_{2}\right] \subset d \text {-simplex }\right\} .
$$

For future algorithms descriptions, we will simply denote $D_{1}(X), \ldots, D_{s_{2}}(X)$ the $s_{2}$ degrees of the $s_{2} 2$-simplices of the complex $X$, with $s_{k}$ being the number of $k$-simplices of $X$.

Next, in order to remove vertices, and not 2-simplices, we need to transmit the superfluousness information of its 2cofaces (2-simplices it is a face of) to a vertex via what is called an index. An index of a vertex is defined to be the minimum of the degrees of the 2-simplices it is a face of. Indeed, a vertex is as sensitive for the coverage as its most sensitive 2 -simplex. The boundary vertices are given a negative index to mark them as unremovable by the algorithm: we do not want the covered area to be shrunk.

Definition 2: The index of a vertex $v$ is the minimum of the degrees of the 2-simplices it is a vertex of:

$$
I[v]=\min \left\{D\left[v_{0}, v_{1} v_{2}\right] \mid v \in\left[v_{0}, v_{1}, v_{2}\right]\right\},
$$

If $v$, a vertex, is a boundary vertex, then $I[v]=-1$.

Finally, the indices give an optimal order for the removal of the vertices: the greater the index of a vertex, the bigger the cluster it is part of, and the more likely it is superfluous for the coverage of its abstract simplicial complex. Therefore, the vertices with the greatest index are candidates for removal: one is chosen randomly. If its removal does not change the homology, i.e. if it does not modify its Betti numbers $\beta_{0}$ and $\beta_{1}$, then it is effectively removed. Otherwise it is flagged as unremovable the same way the boundary vertices are. The algorithm goes on until every remaining vertex is unremovable, thus achieving optimal result.

For more information on the reduction algorithm we refer to $[5]$.

\section{Simulation model}

We want to represent the nodes of a cellular network and its coverage. We are interested in future cellular networks equipped with SON technology, that means cellular networks 
of 4-th and higher generation. For example for a LTE network, its nodes are the eNBs, femtocells, and relays that constitute it. Then we want to compute the network's coverage constituted of coverage disks centered on the nodes. The Čech abstract simplicial complex provides the exact representation of the network's coverage. Its construction for a fixed coverage radius $r$ for all the network's nodes is given:

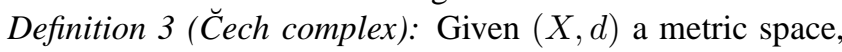
$\omega$ a finite set of points in $X$, and $r$ a real positive number. The $\breve{C}$ ech complex of $\omega$, denoted $\mathcal{C}_{r}(\omega)$, is the abstract simplicial complex whose $k$-simplices correspond to $(k+1)$-tuples of vertices in $\omega$ for which the intersection of the $k+1$ balls of radii $r$ centered at the $k+1$ vertices is non-empty.

However, the Čech complex can be hard to compute, and requires some geographical information that is not always available. For instance Femtocells are not GPS-enabled. There exists an approximation of the coverage Čech complex that is only based on the connectivity information: the so-called neighbor list of each nodes of a SON-capable cellular network. This approximation is the Vietoris-Rips abstract simplicial complex which is defined as follows:

Definition 4 (Vietoris-Rips complex): Given $(X, d)$ a metric space, $\omega$ a finite set of points in $X$, and $r$ a real positive number. The Vietoris-Rips complex of parameter $2 r$ of $\omega$, denoted $\mathcal{R}_{2 r}(\omega)$, is the abstract simplicial complex whose $k$ simplices correspond to unordered $(k+1)$-tuples of vertices in $\omega$ which are pairwise within distance less than $2 r$ of each other.

The definitions of the Čech and the Vietoris-Rips complex of a cellular network can be extended to include different distance parameters in order to represent nodes with different coverage radii. The Čech complex represents the network and its exact topology. However, using the Vietoris-Rips complex representation, it is possible to have so-called triangular holes in the network that do not appear in the complex. The probability of that happening in computed in [29], and in our case where it is upper-bounded by about $0.03 \%$ for a cellular network simulated with a Poisson point process.

In simulations and figures, we can consider either a communication/coverage disk approach, or directly a neighbor list approach. In the first case, any node within the communication disk of a given node is added to its neighbor list, or when two coverage disks of two nodes intersect they are added to each other neighbor lists. Note that communication radii are larger, usually twice as large, than coverage radii. Finally, the abstract simplicial complex of Vietoris-Rips type is then build based on the neighbor lists.

\section{SELF-CONFIGURATION FREQUENCY AUTO-PLANNING ALGORITHM}

\section{A. Problem formulation}

In the frequency planning problem, the topology of the network is not relevant since it is not modified. So we use the simplicial complex representation only for the characterization of clusters without location information, and not for topology computation. First we need to build the abstract simplicial complex representing the cellular network. In future cellular networks, every transmitting nodes (eNBs, Femtocells, relays...) have a neighbor list created and updated with the ANR feature. With this neighbor list information, we can build the abstract simplicial complex representing the network, each node is represented by a 0 -simplex and each neighbor, either 1 -way or 2-way, relationship is represented by a 1 -simplex. The other simplices are then created with only the 1-simplices information (when three 0 -simplices are connected via three 1 -simplices then a 2 -simplex is created, and so on).

The goal of a frequency planning algorithm is to assign frequencies to every network's nodes so that the interference between them is minimum using the smallest number of frequencies possible. In this article, we only consider the one frequency per node case, and co-channel interference, i.e. interference between two nodes using the same frequency. However, the main idea of the algorithm can be extended to several frequencies per node, and interference between different frequencies by considering group of frequencies.

Interference is a two nodes relationship so it can be represented by an interference graph. It is possible to consider any interference model steady through frequencies and time (at least the duration of the configuration). Reliable communication is achievable if the interference is under a chosen threshold. In the interference graph, every network node is represented, then if the interference between two nodes is higher than the threshold, an edge is drawn between them. Consequently, two nodes linked by an edge in the interference graph shall not share the same frequency or the interference level will be too high for reliable communication inside at least one of the two cells.

\section{B. Algorithm description}

We consider a cellular network, nodes and communication radii, as we can see an example in Fig. 1, of which we compute its abstract simplicial complex representation based on the neighbor lists. In Fig. 2, we can see the interference graph on the left. In our example we choose the interference to be only distance-based. In the general case, the only requirement for the interference model is that it can be represented by a graph constant throughout the frequencies and the duration of the configuration. We can see that the configuration of interference of Fig. 2 will need 4 frequencies because of the 4 linked nodes on the left.
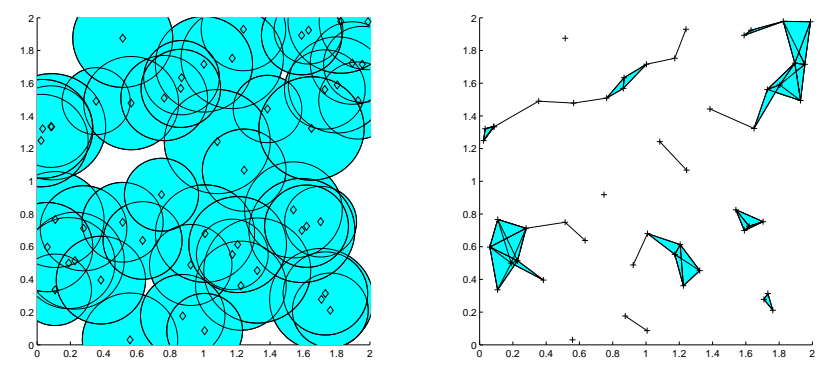

Fig. 1. A cellular network and its abstract simplicial complex representation.

The goal of the algorithm is than to assign a frequency to each node so that no to nodes with interference share 
the same frequency. The algorithm begins by selecting the nodes that will receive the first frequency available. To do that, we apply a modified version of the reduction algorithm presented in Section III. It "removes" nodes until obtaining a interference-free configuration of nodes that receive the first frequency. The order in which the nodes are removed is still decided by the indices but the stopping condition is not the same as in simple reduction. Instead of stopping when the maximum index among every remaining nodes is below a given number, the algorithm stops when there is no more pair of nodes connected to one another in the interference graph. The resulting nodes of the reduction algorithm are then assigned the first frequency and put aside for the remaining of the algorithm.

All the previously "removed" nodes are then collected, and the corresponding abstract simplicial complex recovered. This complex is a subset of the initial complex so there is no need to build another one from scratch. The next step is then to reapply the modified reduction algorithm to this recovered complex to obtain a second set of nodes to which we assign the second frequency. The algorithm goes on until every node has an assigned frequency.

At the end, we have a frequency assigned to every node. We ensured that no two nodes sharing the same frequency will be too close to each other: interference will be under a given threshold. Moreover, the use of our coverage reduction algorithm with the optimized order for nodes removal allows us to obtain a homogeneous usage of every frequency.

The frequency planning scheme obtained by our algorithm for the configuration of Fig. 1 is represented in Fig. 2 on the right. A different color represent a different frequency. We can see that our algorithm has planned four frequencies (black, red, green and blue) of which we can see the communication area for each one in Fig. 3.
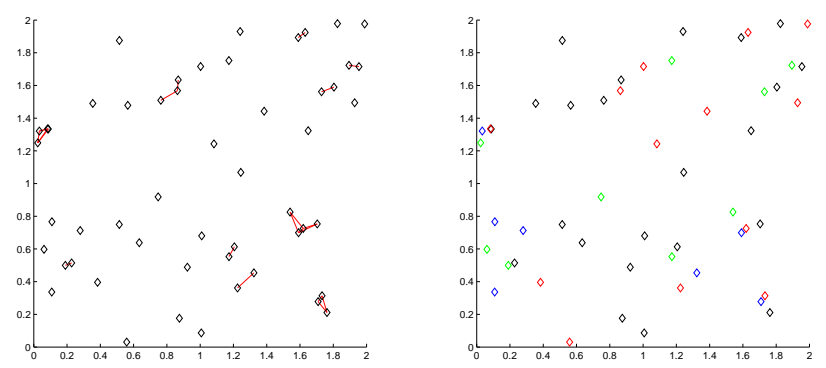

Fig. 2. Interference graph and frequency planning scheme.

We give in Algorithm 1 the full frequency auto-planning algorithm. It requires the set of nodes $\omega$, and the neighbor lists $L_{n}(v)$ for each node $v$ in $\omega$ to build the abstract simplicial complex. If instead of the neighbor lists, one considers the communication radii $r$, then the abstract simplicial complex is the Vietoris-Rips complex $\mathcal{R}_{2 r}(\omega)$. Plus, we need to build the interference graph, so we consider an interference list $L_{i}(v)$ for each node $v$ that contains the list of nodes $v$ has interference with. Then the algorithm returns the list of assigned frequencies for every node of $\omega$.
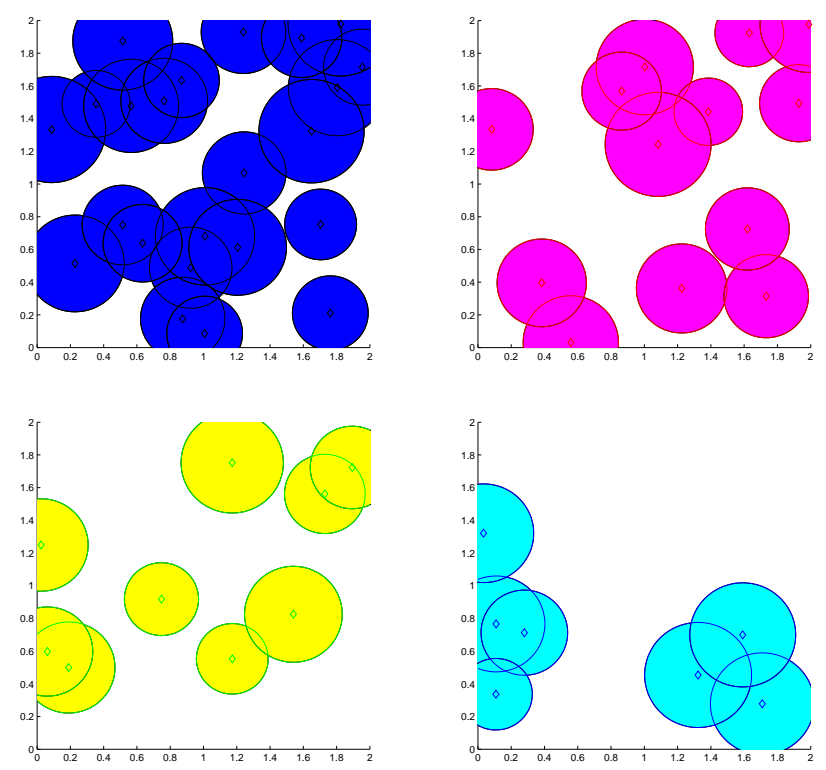

Fig. 3. Coverage for each frequency.

We introduce three parameters in the algorithm description that we define here for a better understanding. First, $N_{\text {assigned }}$ is the number of nodes to which a frequency is assigned. Then, $\mathcal{I}$ is a binary number that is equal to one if there are at least one node with potentially the same frequency than another node in its interference list and zero otherwise. Finally, $f(v)$ is the frequency assigned to the vertex $v \in \omega$.

For the simplicial complex $X$, we denote $s_{k}$ the number of its $k$-simplices, and $v_{1}, \ldots, v_{s_{0}}$ its $s_{0}$ vertices. We do not detail the computations of the degrees $D_{1}(X), \ldots, D_{s_{2}}(X)$ of the 2-simplices and the indices $I\left[v_{1}(X)\right], \ldots, I\left[v_{s_{0}}(X)\right]$ which are explained in [5].

\section{Simulation and complexity}

First, in simulations, we create the set of nodes with a Poisson point process of intensity $\lambda=12$ on a square of side $a=2$. Then we draw a communication radius for every node uniformly between $a / 10$ and $2 / \sqrt{\pi \lambda}$ and we build the Vietoris-Rips complex. The complexity of building the complex is in $O\left(N^{C}\right)$, where $N$ is the number of nodes and $C$ is the clique number, i.e. the size of the largest simplex in the complex. If communication radii are equal to a given $r$, then $C$ is upper-bounded by $N\left(\frac{r}{a}\right)^{2}$ in the general case. This bound can be improved depending on the percolation regimes, see [30]. We compute here worst-case complexities in order to have upper bounds for the complexity of building a simplicial complex, then for the complexity of our algorithms. In large scale networks, the simplicial complex representing the network can be build and stored once at the deployment, then the addition or deletion of some nodes can be done without re-building the complex entirely, with less complexity.

We want to create distance-based interference depending on the different communication radii of the nodes, so we introduce a so-called "rejection" radius. Then, each node has 


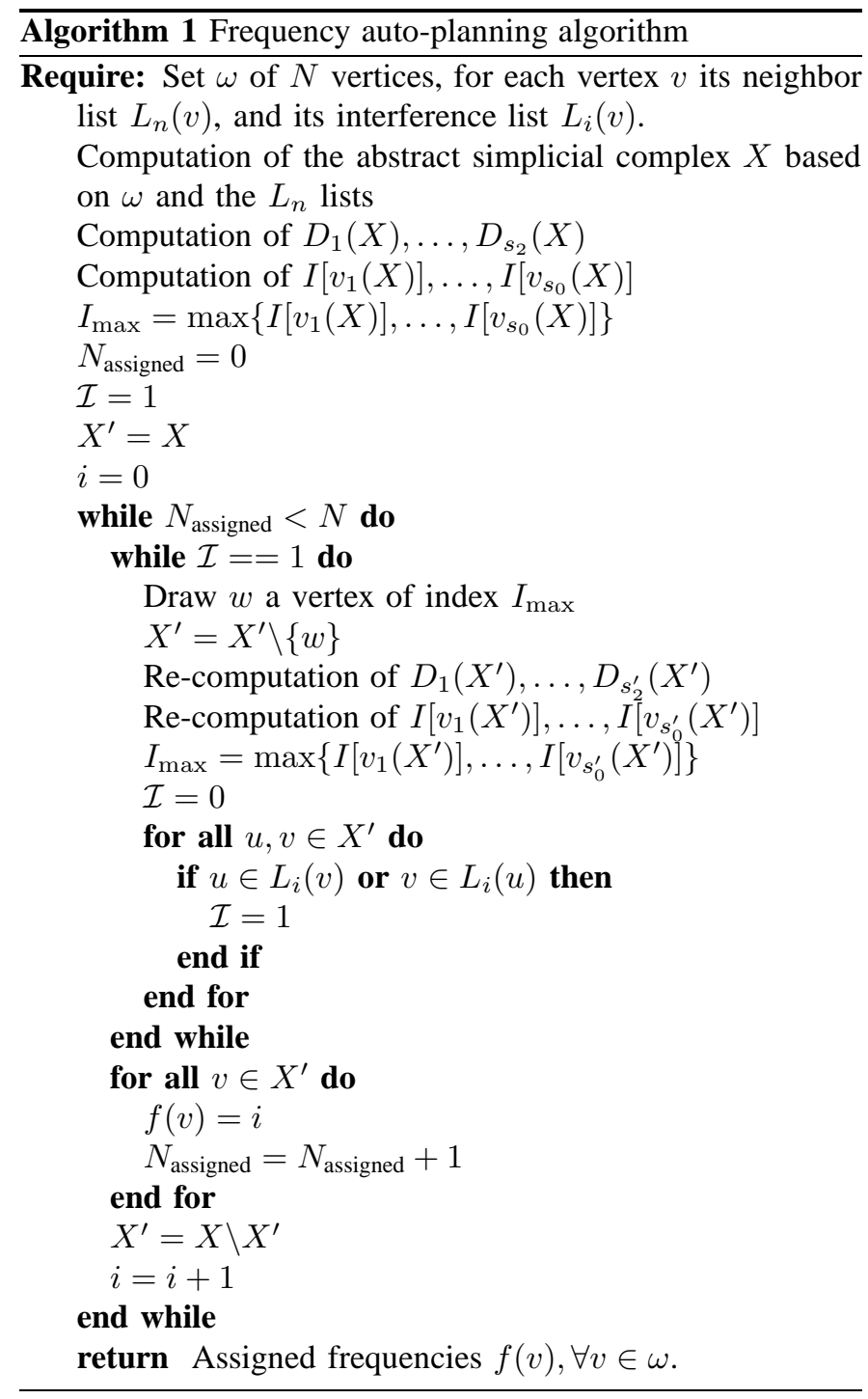

a rejection radius that is a growing function of its communication radius. In simulations, the rejection radii are equal to half their corresponding communication radii. If a given node is inside the disk defined by the rejection radius of another node, then it appears in its interference list and they are linked by an edge in the interference graph. The complexity of building the interference graph is negligible compared to the complexity of building the abstract simplicial complex.

Then, the complexity of the frequency auto-planning algorithm is upper-bounded by the complexity of the reduction algorithm that is called $N_{f}$ times, if $N_{f}$ is the number of assigned frequencies. The complexity of the reduction algorithm is $N s_{2}\left(N+\sum_{k=3}^{C-1} s_{k}\right)$ in the general case according to [5]. However it can be simplified when the set of nodes is drawn uniformly, as in a Poisson point process, on a square, the communication radii are all equal to a given $r$, and the complex is a Vietoris-Rips one. In this case, the complexity is in $O\left(\left(1+\left(\frac{r}{a}\right)^{2}\right)^{N}\right)$, see [5]. Therefore the final complexity of our algorithm is in $O\left(N_{f}\left(1+\left(\frac{r}{a}\right)^{2}\right)^{N}\right)$.

We can see that the complexity of our algorithm is dependent highly on the choice of the simplicial complex repre- sentation. But this representation is needed if one wants to compute the topology of a network, and obtain clustering information without location information. We do not compare the complexity of our homology-based algorithm to classic graph-based algorithms since the homology parameters we encounter such as the radius $r$ are not relevant in a graphbased approach.

\section{Performance comparison}

In this section, we compare the performance of our frequency auto-planning algorithm to the greedy coloring algorithm. We choose this algorithm because our algorithm, as well as the reduction algorithm, and consequently every algorithm proposed in this article is of greedy type. Thus we compare two greedy algorithms, ours is simplicial homology-based, while the coloring algorithm is graph-based.

The frequency planning can be viewed as a graph coloring problem. If one considers the interference graph, then the optimal number of frequencies to assign is the chromatic number of the interference graph. The greedy coloring algorithm provides a coloring assigning the first new color available for each node. Therefore, the greedy coloring algorithm is a frequency planning algorithm. And the number of frequencies planned is at most the maximum node degree of the interference graph plus one. The greedy coloring gives especially good results for sparse graphs as the interference graph is. So the first parameter that we will use to compare the performance of both algorithm is the number of planned frequencies.

For each realization of the Poisson process, we compute the number of frequencies planned by the greedy coloring algorithm that we denote $N_{g}$. Then on all realizations with a given $N_{g}$, we compute the mean number of frequencies, denoted $N_{f}$, planned by our algorithm, so we can see the difference between the two algorithms. We also indicate which percentage of the simulations these scenarios are, the occurrence is added for statistical information. The results are obtained in mean over $10^{4}$ configurations.

\begin{tabular}{|c|cccccc|}
\hline \multicolumn{2}{|c}{$N_{g}$} & 2 & 3 & 4 & 5 & 6 \\
\hline $\mathbf{E}$ & $N_{f} \mid N_{g}$ & 4.00 & 5.04 & 5.83 & 6.43 & 7.13 \\
\hline \multicolumn{2}{|c|}{ Occurrence } & $8.8 \%$ & $55.6 \%$ & $29.4 \%$ & $5.3 \%$ & $0.7 \%$ \\
\hline
\end{tabular}

TABLE I

MEAN NUMBER OF PLANNED FREQUENCIES.

In Table I, we can see the mean number of frequencies planned by our algorithm given the number of frequencies planned by the greedy coloring algorithm. We can see that there is a difference between the two solutions: it is not negligible in the beginning, but it decreases with the number of frequencies. Thus, our algorithm reaches its optimal performance when the number of frequencies grows for the same mean number of nodes, that is to say when there are clusters of nodes.

However, even if the greedy coloring algorithm fares well in the number of planned frequency, it leads to a disparate utilization of frequencies. Indeed, if there is only one clique of maximum size, one frequency will be only used for one 
node of this clique, and for no other node in the whole configuration. Therefore, the greedy coloring algorithm give good results for a homogeneous network, but not for a cluster network for example. For an optimal utilization of frequencies, each frequency should cover the whole area, but it is not always achievable if there are not enough nodes to cover several times the whole area. Our algorithm aims at a more homogeneous utilization of each ressource. In order to show that, we compare the percentage of area covered by each frequency on the total covered area for our frequency autoplanning algorithm and for the greedy coloring algorithm. We consider an area to be covered by a frequency if it is inside the communication disk of a node using this frequency. The percentages are given in mean over $10^{4}$ simulations using the same setting as in the first comparison.

Greedy coloring algorithm
\begin{tabular}{|ccccc|}
\hline$N_{g}$ & 3 & 4 & 5 & 6 \\
\hline$f_{1}$ & $97.3 \%$ & $97.0 \%$ & $96.7 \%$ & $96.3 \%$ \\
\hline$f_{2}$ & $47.6 \%$ & $50.1 \%$ & $49.9 \%$ & $51.1 \%$ \\
\hline$f_{3}$ & $12.9 \%$ & $17.7 \%$ & $18.8 \%$ & $20.2 \%$ \\
\hline$f_{4}$ & & $7.2 \%$ & $8.8 \%$ & $8.5 \%$ \\
\hline$f_{5}$ & & & $6.2 \%$ & $6.7 \%$ \\
\hline$f_{6}$ & & & & $6.2 \%$ \\
\hline Occurrence & $55.6 \%$ & $29.4 \%$ & $5.3 \%$ & $0.7 \%$ \\
\hline
\end{tabular}

Frequency auto-planning algorithm
\begin{tabular}{|ccccc|}
\hline$N_{f}$ & 3 & 4 & 5 & 6 \\
\hline$f_{1}$ & $71.3 \%$ & $62.1 \%$ & $54.2 \%$ & $49.2 \%$ \\
\hline$f_{2}$ & $62.3 \%$ & $56.4 \%$ & $51.7 \%$ & $47.4 \%$ \\
\hline$f_{3}$ & $35.6 \%$ & $46.4 \%$ & $45.7 \%$ & $42.9 \%$ \\
\hline$f_{4}$ & & $24.3 \%$ & $35.6 \%$ & $37.8 \%$ \\
\hline$f_{5}$ & & $18.8 \%$ & $28.1 \%$ \\
\hline$f_{6}$ & & & $15.3 \%$ \\
\hline Occurrence & $7.0 \%$ & $23.0 \%$ & $29.4 \%$ & $22.6 \%$ \\
\hline
\end{tabular}

TABLE II

MEAN PERCENTAGE OF COVERED AREA FOR EACH FREQUENCY

We can see in Table II the percentage of area covered by each frequency planned by our algorithm and the greedy coloring algorithm. The results are presented depending on the number of planned frequencies, we also indicate the number of simulations these results concern for statistical relevance. For our algorithm, even if the percentage decreases with the order in which the frequencies are planned, which is logical since the first frequency is planned in first and so on, we can see that a rather homogeneous coverage is provided. Doing that, our algorithm maximizes the usage of each resource. We can see that for the greedy coloring algorithm, the frequencies are not used equally: the first two frequencies are always a lot more planned than the other ones, the latter are thus under-used.

\section{SELF-OPTIMIZATION ENERGY CONSERVATION ALGORITHM}

\section{A. Problem formulation}

We are now interested in the self-optimization of a cellular network previously configured. Indeed, during off-peak hours a cellular network is under-used, we propose an algorithm that aims at reducing the energy consumption when user traffic is reduced. First we want to represent the considered cellular network and its topology with an abstract simplicial complex.
The network is constituted of transmitting nodes and their associated coverage disks. In future cellular networks, these coverage disks can vary in size by modifying the configuration parameters of the base stations. We choose to consider here the maximum size of these disks in order to maximize the coverage of each node so that a maximum number of nodes can be switched-off. Maximizing the coverage disk of one node induces maximizing the size of its neighbor list. Then we build the abstract simplicial complex representing the network and its maximum neighbor lists. After that, we have to consider boundary nodes differently from the other ones. Indeed, these nodes allow us to delimit the area to cover.

Finally we have to define how we represent user traffic in the cellular network. In our simulations, we choose to create groups of network nodes. Then for each group of nodes, the required quality of service (QoS) is a given number of nodes that are required to stay on in order to satisfy the traffic of the users inside that group coverage. Therefore, every group of nodes has a required minimum size. This QoS metric is quite artificial, but our algorithm can take into account any QoS as long as it is defined in terms of required number of ressources from a given pool of ressources available to a given group of nodes. We choose to implement this simplistic metric because it is the easiest one to implement. Other metrics can be considered, the only rule being that one node must pertain to exactly one group.

\section{B. Algorithm description}

We consider a cellular network with transmitting nodes and their maximal coverage radii that we represent with an abstract simplicial complex. We can see an example of network and its complex associated in Fig. 4. The boundary nodes are computed here via the convex hull and are in red in the figure.
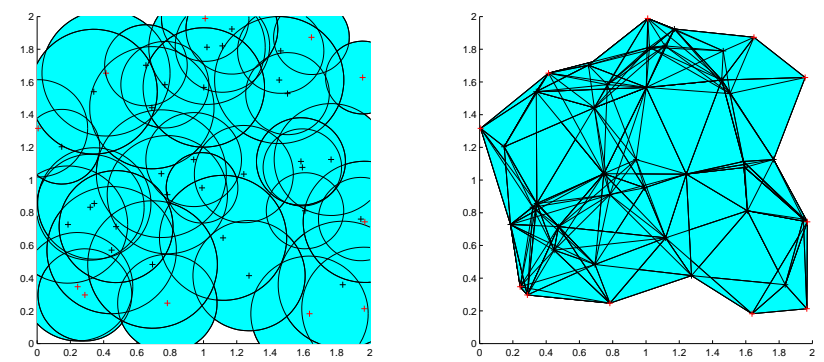

Fig. 4. A cellular network and its abstract simplicial complex representation.

Then for the configuration of Fig. 4, we represent the QoS groups of nodes in different colors, and give a table with their corresponding size and required QoS in Fig. 5 in the figure on the left.

The algorithm then begins by the computation of the Betti numbers. They characterize the topology of the network that we do not want to modify: the number of connected components and the number of coverage holes. In the example of Fig. 4, there is one connected component, $\beta_{0}=1$, and no coverage hole, $\beta_{1}=0$. Then the algorithm aims at reducing 
the number of nodes as the reduction algorithm presented in Section III, but we want to keep enough nodes to satisfy the traffic represented by a minimum number of nodes to keep on by group. So we apply a modified reduction algorithm with a different breaking point. Instead of stopping when the area is covered by a minimum number of nodes, the algorithm stops when each group of nodes has been reduced to the size of its required QoS. We can see the obtained result for the configuration of Fig. 4 in Figure 5 on the right. The kept nodes are circled.
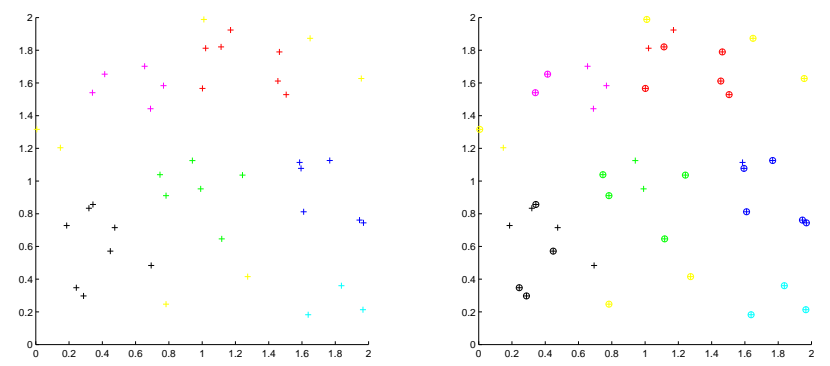

\begin{tabular}{|ccccccccccc|}
\hline Groups & 1 & 2 & 3 & 4 & 5 & 6 & 7 & 8 & 9 & 10 \\
\hline Size & 8 & 7 & 6 & 6 & 5 & 3 & 2 & 2 & 2 & 1 \\
\hline QoS & 3 & 5 & 5 & 4 & 2 & 1 & 1 & 1 & 2 & 1 \\
\hline
\end{tabular}

Fig. 5. QoS groups and required QoS.

The cellular network and its abstract simplicial complex representation is represented in Fig. 6.
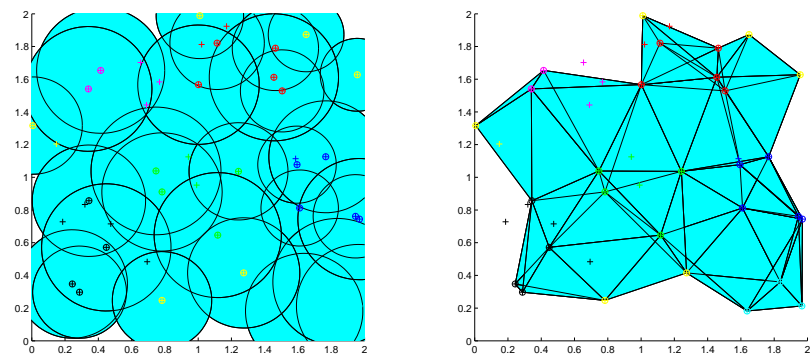

Fig. 6. The network after the first step.

Finally, given this configuration of switched-on nodes, the algorithm tries to reduce as much as possible the coverage radii of each node without creating a coverage hole to minimize the energy consumption. In Fig. 7, we can see the final configuration of the cellular network with the optimized coverage radii and its abstract simplicial complex representation for the configuration of Fig. 4.

In the end, we have a configuration of nodes to keep switched-on that is optimal. From the first part of the algorithm, we ensure that enough nodes are kept on to satisfy the QoS representing user traffic. Then, in the second part of the algorithm, we ensure that no energy is spend uselessly by optimizing the size of the serving cells.

We give in Algorithm 2 the full energy conservation algorithm. It requires the set of nodes $\omega$ and their neighbor lists in order to build the abstract simplicial complex. If instead of the neighbor lists, one considers the communication radii $r$, then
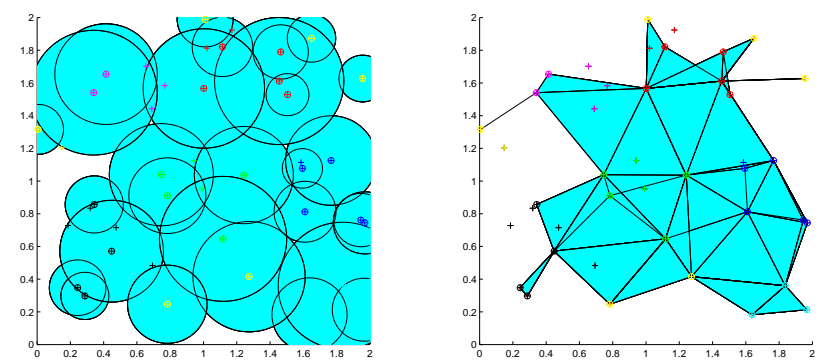

Fig. 7. Final configuration.

the abstract simplicial complex is the Vietoris-Rips complex $\mathcal{R}_{2 r}(\omega)$. Or if one wants to represent the exact topology and considers the coverage radii $r$, then it is the Cech complex $\mathcal{C}_{r}(\omega)$.

The groups are represented by the variable $G(v)$ that for each node $v$ gives its group number. Then for a given group $g, Q(g)$ is the minimum number of nodes required by the QoS, while $S(g)$ is its number of nodes. So we always have $Q(g) \leq S(g)$. Either these parameters have to be given as input, or the way to compute them is to be integrated into the algorithm. We can see that the breaking point of the "while" loop of the algorithm takes the QoS parameter $Q$ into account. Then a node can be removed if and only if it does not modify the Betti numbers of the abstract simplicial complex, and it is not needed for the QoS requirements.

Optional coverage radii reduction for each node is done in the last loop. To implement that last step, maximum coverage radii are needed in input. The order in which the coverage radii are examined is random. The reduction can be made by steps for example. At the end, the algorithm returns the list of kept nodes with their new coverage radii.

\section{Simulation and complexity}

As in the previous section, we simulate the set of nodes with a Poisson point process on a square of side $a=2$. However, since we need boundary nodes and do not need a great number to see the efficiency of the algorithm, the intensity of the Poisson point process is lowered to $\lambda=6$. Then the coverage radii of the vertices from the Poisson point process are sampled uniformly between $a / 10$ and $2 / \sqrt{\pi \lambda}$. And the coverage radii of the boundary nodes is set to $a / 3$. The complexity of building the Vietoris-Rips complex is still in $O\left(N^{N\left(\frac{r}{a}\right)^{2}}\right)$, where $N$ is the number of vertices and $r$ is the common coverage radius.

To create the groups of nodes in simulations, we have to take some care. Indeed, these groups have to make sense geographically, but we do not have location information. So in order to consider clusters of nodes, every group is defined by a maximum simplex, a simplex which is not the face of any other simplex. The groups are created so that every node pertains to exactly one group. Then, for every group $g$ of size $S(g)=k$, an integer is uniformly drawn between 1 and $k$, this integer is then the required number of nodes to keep on, i.e. $Q(g)$. 


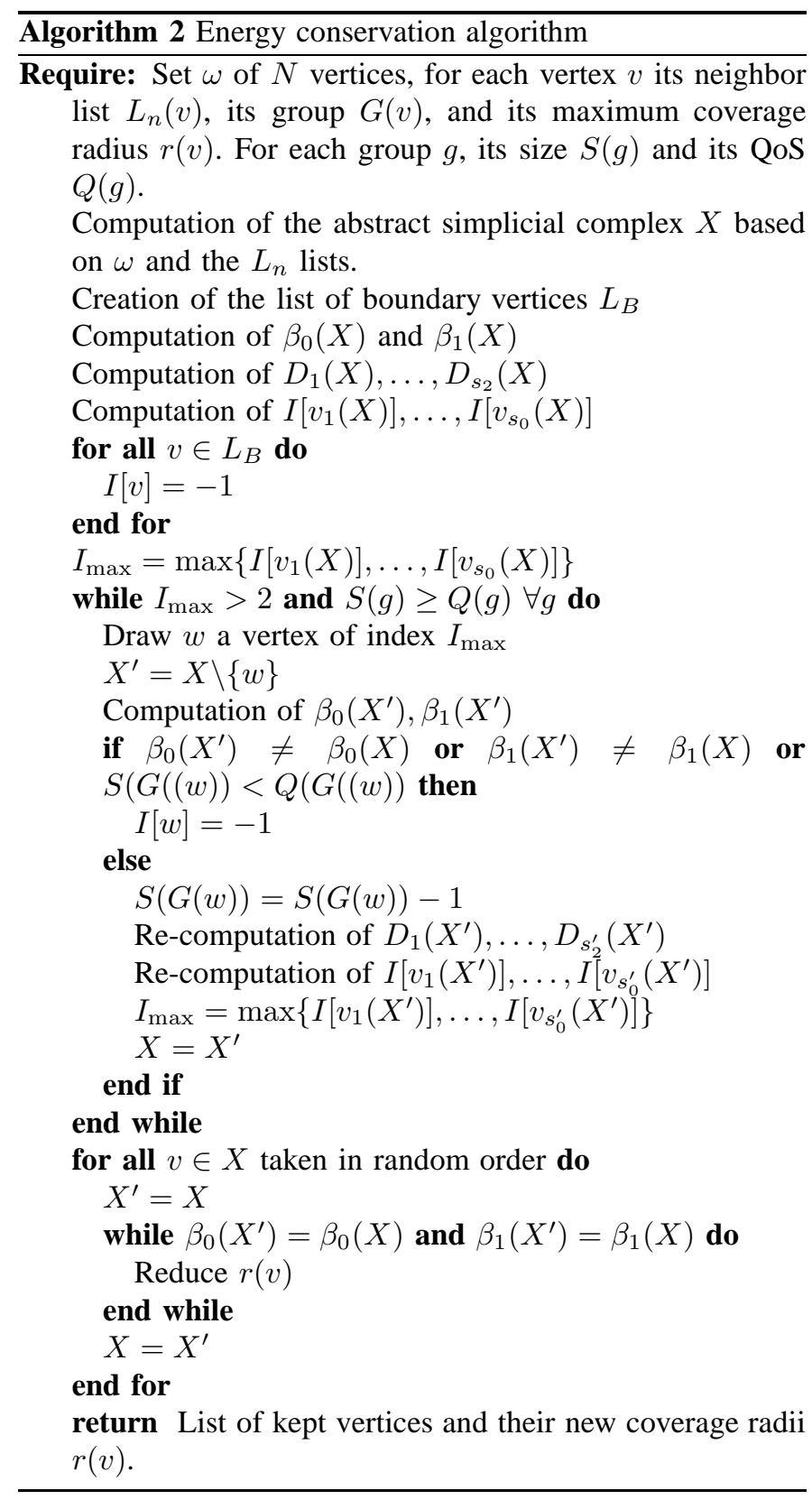

For our simulations, we choose to give advantage to the larger simplices for the constitution of the groups. Thus, the first group will consist of the largest simplex, or one randomly chosen among the largest ones, then simplices of smaller size will become groups until every node is part of a group. It is possible to consider other rules for the constitution of the groups, but it has to follow one condition: every node must pertain to exactly one group. The constitution of $N_{G}$ groups is given in Algorithm 3.

The complexity of the energy conservation algorithm is the same as the reduction algorithm since it is an improved reduction algorithm, that is $O\left(\left(1+\left(\frac{r}{a}\right)^{2}\right)^{N}\right)$, see [5].

\section{Performance comparison}

In this section, we compare the performance of our algorithm to an optimal, not always achievable greedy solution.

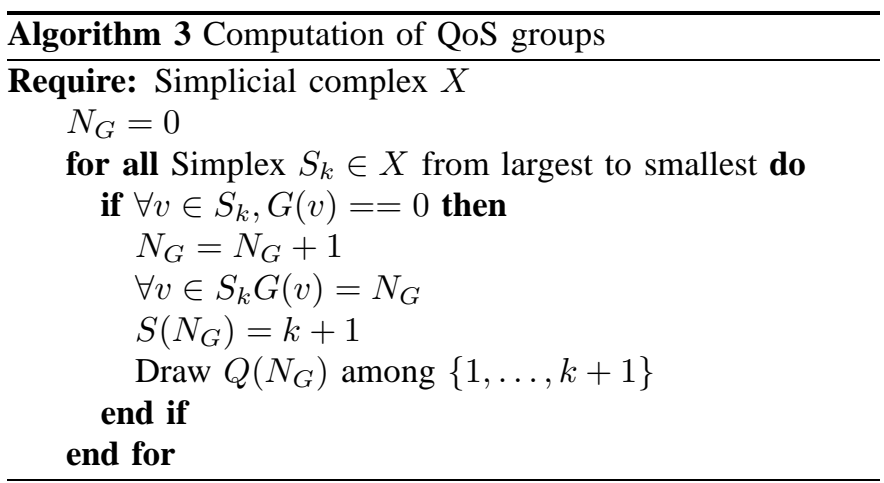

As in the previous section we choose to compare our greedy simplicial homology-based energy conservation algorithm to a greedy graph-based algorithm. We do not know of a energy conservation algorithm that can switch-off nodes during offpeak hours while maintaining coverage. Thus, we compare the number of switched-on nodes after the execution of our energy conservation algorithm, to the number of nodes needed for the QoS, given by the sum of the $Q(g)$ for all $g$. It is important to note that this optimal solution is not always achievable because it does not take into account that the area is to stay covered. Some nodes have to be kept for traffic reasons, while other are kept to maintain connectivity and/or coverage. This number of nodes can not be obtained for a random configuration of nodes whose positions do not follow a given pattern.

Our simulation results are computed on $10^{4}$ configurations of We denote by $N_{o}=\sum_{g} Q(g)$ the optimal number of kept nodes for traffic reasons, and by $N_{k}$ the number of kept nodes with our energy conservation algorithm for both traffic and coverage reasons. First we compute the percentage of simulations for which we have a given difference between the obtained number $N_{k}$ and the minimum number $N_{o}$ of kept nodes.

\begin{tabular}{|cccccc|}
\hline$N_{k}-N_{o}$ & 0 & 1 & 2 & 3 & 4 \\
\hline Occurrence & $1.3 \%$ & $4.2 \%$ & $8.5 \%$ & $12.5 \%$ & $15.4 \%$ \\
\hline \hline 5 & 6 & 7 & 8 & 9 & $\geq 10$ \\
\hline $15.4 \%$ & $14.3 \%$ & $11.0 \%$ & $7.5 \%$ & $4.9 \%$ & $4.7 \%$ \\
\hline
\end{tabular}

TABLE III

OCCURRENCES OF GIVEN DIFFERENCES BETWEEN $N_{k}$ AND $N_{o}$.

We can see in Table III the percentage of simulations in which the number of kept nodes is different from the optimal number of nodes. For $1.3 \%$ of the simulations the optimal number is reached. In $82.8 \%$ of our simulations the difference between the optimal and the effective number of kept nodes is smaller than 7 , and it never exceeds 18 . From our simulations, an average of $N_{k}-N_{o}=5.16$ nodes are kept by our algorithm only for coverage reasons, and not for traffic reasons. To explain this number, we can note that the 16 boundary nodes are always kept for coverage reasons, even if they can also be used for traffic reasons depending on the configurations.

To have more advanced comparison, for the $10^{4}$ configurations, we compute the optimal number of nodes. Then for each optimal number of nodes $N_{o}$ that occurred the most, we compute the mean number of kept nodes $\mathbf{E}\left[N_{k} \mid N_{o}\right]$ over the 
simulations which have $N_{o}$ for optimal number. The results are given in Table IV. For comparison, we also compute the difference between $N_{o}$ and $N_{k}$ in percent. We finally indicate which percent of our $10^{4}$ simulations these cases occur to show the relevance of these statistical results.

\begin{tabular}{|c|c|c|c|c|c|}
\hline$N_{o}$ & 22 & 23 & 24 & 25 & 26 \\
\hline $\begin{array}{l}\mathbf{E}\left[N_{k} \mid N_{o}\right] \\
\end{array}$ & 28.95 & 29.52 & 30.04 & 30.69 & 31.30 \\
\hline Difference & $31.6 \%$ & $28.4 \%$ & $25.1 \%$ & $22.8 \%$ & $20.4 \%$ \\
\hline Occurrence & $5.0 \%$ & $6.2 \%$ & $7.0 \%$ & $7.4 \%$ & $7.9 \%$ \\
\hline 27 & 28 & 29 & 30 & 31 & 32 \\
\hline 31.85 & 32.68 & 33.25 & 33.95 & 34.64 & 35.38 \\
\hline $18.0 \%$ & $16.7 \%$ & $14.7 \%$ & $13.1 \%$ & $11.7 \%$ & $10.6 \%$ \\
\hline $7.4 \%$ & $7.5 \%$ & $7.2 \%$ & $6.3 \%$ & $5.9 \%$ & $4.8 \%$ \\
\hline
\end{tabular}

We can see in Table IV that the more nodes are needed, the less difference there is between the number of kept nodes for both coverage and traffic reasons $N_{k}$ and the optimal minimum number of nodes kept for only traffic reasons $N_{o}$. Indeed, if more nodes are needed for traffic, there is a great chance that these nodes can cover the whole area, and fewer nodes are needed only for coverage reasons.

\section{DISASTER RECOVERY ALGORITHM}

In this section we present a disaster recovery algorithm introduced in [6] of which we remind the main idea and investigate more thoroughly the performance in the next section. But first, we need to introduce some stochastic geometry concepts.

\section{A. Repulsive point processes}

In this section, we aim at repairing a damaged cellular network by adding new nodes randomly in the damaged area. The most common point process in cellular network representation is the Poisson point process. However in this process, conditionally to the number of points, their positions are independent from each other. This independence creates some aggregations of points, as well as voids (i.e. coverage holes), both of which are not convenient for the recovery of a network. That is why we propose the use of determinantal point processes, in which the points positions are not independent anymore.

General point processes can be characterized by their socalled Papangelou intensity. Informally speaking, for $x$ a location, and $\omega$ a realization of a given point process, that is a set of points, $c(x, \omega)$ is the probability to have a point in an infinitesimal region around $x$ knowing the set of points $\omega$. For Poisson process, $c(x, \omega)=1$ for any $x$ and any $\omega$. A point process is said to be repulsive (resp. attractive) whenever $c(x, \omega) \geq c(x, \zeta)$ (resp. $c(x, \omega) \leq c(x, \zeta))$ as soon as $\omega \subset \zeta$. For repulsive point process, that means that the greater the set of points, the smaller the probability to have an other point.

Among repulsive point processes, we are in particular interested in determinantal processes:

Definition 5 (Determinantal point process): Given $X \quad$ a Polish space equipped with the Radon measure $\mu$, and
$K$ a measurable complex function on $X^{2}$, we say that $N$ is a determinantal point process on $X$ with kernel $K$ if it is a point process on $X$ with correlation functions $\rho_{n}\left(x_{1}, \ldots, x_{n}\right)=\operatorname{det}\left(K\left(x_{i}, x_{j}\right)_{1 \leq i, j \leq n}\right)$ for every $n \geq 1$ and $x_{1}, \ldots, x_{n} \in X$.

We can see that when two points $x_{i}$ and $x_{j}$ tend to be close to each other for $i \neq j$, the determinant tends to zero, and so does the correlation function. That means that the points of $N$ repel each other. There exist as many determinantal point processes as functions $K$. We are interested in the following:

Definition 6 (Ginibre point process): The Ginibre point process is the determinantal point process with kernel $K(x, y)=\sum_{k=1}^{\infty} B_{k} \phi_{k}(x) \overline{\phi_{k}(y)}$, where $B_{k}, k=1,2, \ldots$, are $k$ independent Bernoulli variables and $\phi_{k}(x)=\frac{1}{\sqrt{\pi k !}} e^{\frac{-|x|^{2}}{2}} x^{k}$ for $x \in \mathbb{C}$ and $k \in \mathbb{N}$.

The Ginibre point process is invariant with respect to translations and rotations, making it relatively easy to simulate on a compact set. Moreover, the repulsion induced by a Ginibre point process is of electrostatic type. The principle behind the repulsion lies in the probability density used to draw points positions. The probability to draw a point at the exact same position of an already drawn point is zero. Then, the probability increases with increasing distance from every existing points. Therefore the probability to draw a point is greater in areas the furthest away from every existing points, that is to say in coverage holes. The simulation of Ginibre determinantal point processes is detailed in [31].

\section{B. Main idea}

The disaster recovery algorithm aims at restoring a damaged cellular network. Thus, we consider a cellular network presenting coverage holes and possibly many disconnected components. The Čech complex or its approximation the Vietoris-Rips complex is build based on the set of nodes and either their coverage or communication radii, or their neighbor lists. We also need a list of boundary nodes, which can be fictional, in order to know the whole area to be covered once the network is repaired.

In this section we suppose that the node locations are known. Indeed the disaster recovery algorithm provides the locations where to put new nodes in order to patch the network. We also restrict ourselves to a fixed common coverage radius for every nodes, even if the main idea can be extended. We can see an example of damaged cellular network with a square boundary of fictional nodes and its representation by the approximated coverage complex: the Vietoris-Rips complex in Fig. 8.

The algorithm then adds new nodes in addition to the set of existing nodes. For the addition of nodes, several methods, deterministic or random, are possible. But we choose here to focus here on the most fitted random method: determinantal point processes. However, the number of new added nodes is not computed as in determinantal point processes, but via an incrementation. It is first set to the minimum number of nodes to cover the whole area minus the number of existing nodes. When the nodes are added, the two first Betti numbers are computed. Then if there are more than one connected 

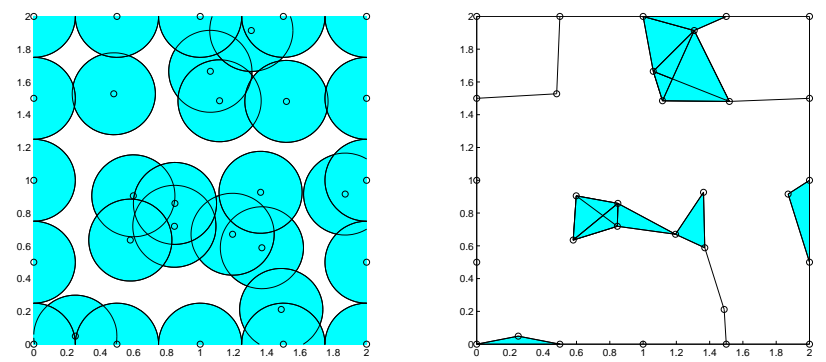

Fig. 8. A damaged cellular network with a fixed boundary.

component, or any coverage hole, other new nodes are added. Their number is incremented with a random variable following an exponential growth: first set to 1 , it is doubled every time new nodes are added and the network is still not patched.

Using determinantal point processes for the addition of nodes allows us to not only take into account the number of existing nodes via the computation of the number of added nodes, but also their locations. Indeed, the existing nodes are considered part of the point process, then new nodes positions are drawn following a determinantal point process. The addition of new nodes stops as soon as the network is repaired: one connected component and no coverage hole.

We can see the first step of the disaster recovery algorithm illustrated in Fig. 9 for the cellular network of Fig. 8. Existing nodes are black circles while added nodes are red plusses.
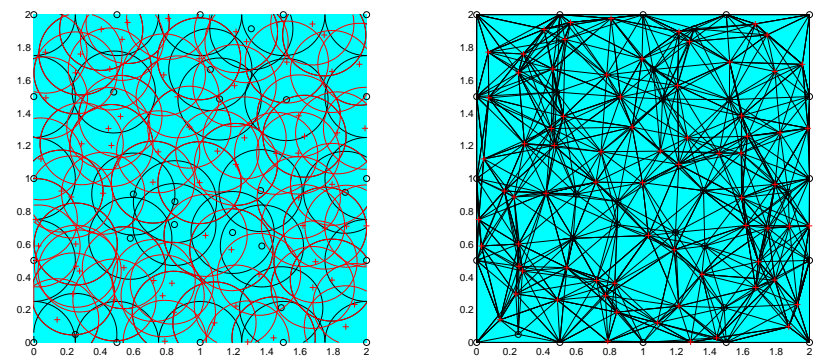

Fig. 9. The repaired network.

Finally, the next step of our approach is to run the reduction algorithm presented in Section III which maintains the topology of the repaired cellular network. At this step, we remove some of the new nodes we just virtually added in order to achieve an optimal result with a minimum number of really added nodes. We can see in Fig. 10 an execution of the reduction algorithm on the intermediate configuration of Figure 9 which constitutes of the second and final step of the disaster recovery algorithm. Removed nodes are represented by blue diamonds.

We give in Algorithm 4 the outline of the algorithm. The algorithm requires the set of $N_{i}$ initial nodes $\omega_{i}$, the fixed coverage radius $r$, as well as the list of boundary nodes $L_{b}$.

\section{Simulation and complexity}

The set of nodes is no more simulated by a Poisson point process, since we want to simulate a damaged cellular network
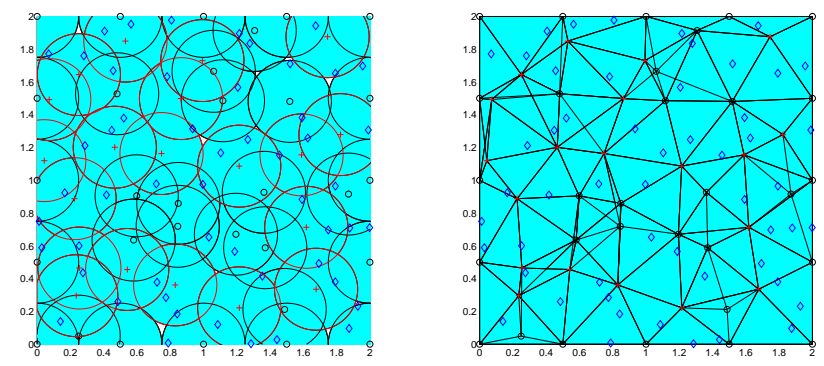

Fig. 10. Final configuration.

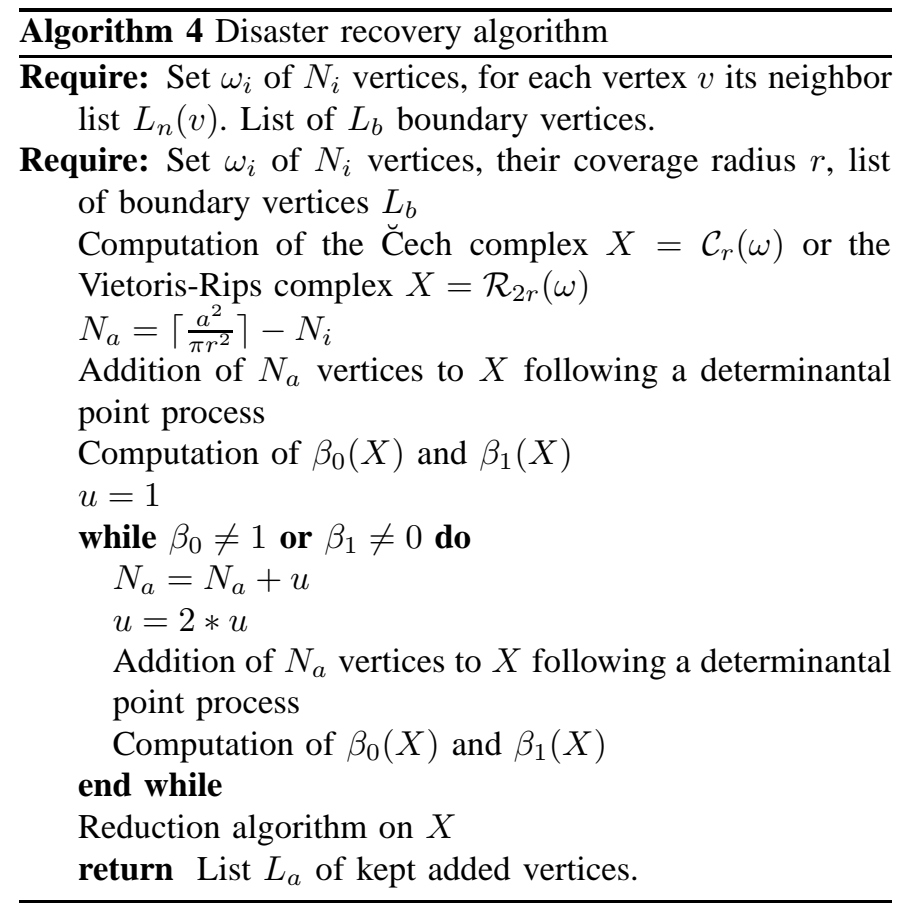

at different stages of damage. Every configuration is still simulated on a square of side $a=2$, and nodes have a common coverage radius of $r=0.5$ for the construction of the VietorisRips complex, of which complexity is unchanged $O\left(N^{N\left(\frac{r}{a}{ }^{2}\right)}\right)$.

The complexity of the drawing of nodes following a determinantal point process is upper-bounded by the one of the reduction algorithm, see [31] for details about the simulation of Ginibre determinantal point processes. So the complexity of the disaster recovery algorithm is in $O\left(\left(1+\left(\frac{r}{a}\right)^{2}\right)^{N}\right)$. It is important to note that the number of nodes $N$ is the sum of the number of initial nodes $N_{i}$ and added nodes $N_{a}$.

\section{Performance comparison}

We now compare the performance results of our disaster recovery algorithm to the best known greedy coverage recovery algorithm: the greedy algorithm for the set cover problem. As the reduction algorithm, our homology-based disaster recovery algorithm is of greedy type, so we compare it to a graph based greedy algorithm. The only way to obtain coverage with graph representation is to lay nodes following a given pattern, that is what the greedy algorithm for the set cover problem does, with a square pattern. It lays a grid of potential new nodes, 
and adds first the node the furthest from every other existing node, and so on until the furthest node is in the coverage of an existing or added node.

We first compare the mean number of added nodes following four different scenarios for the initial state. Indeed, the performance of a recovery algorithm can be really affected depending on how damaged is the cellular network to repair. So the different scenarios are defined by the mean percentage of covered area before running a recovery algorithm. In Table $\mathrm{V}$, we present the mean final number of added nodes for the set cover algorithm and our homology algorithm over $10^{4}$ configurations for each scenario.

\begin{tabular}{|ccccc|}
\hline$\%$ of area initially covered & $20 \%$ & $40 \%$ & $60 \%$ & $80 \%$ \\
\hline Set Cover algorithm & 3.65 & 3.36 & 2.82 & 1.84 \\
\hline Homology algorithm & 4.47 & 3.85 & 2.98 & 1.77 \\
\hline TABLE V \\
MEAN FINAL NUMBER OF ADDED NODES E $\left[N_{f}\right]$
\end{tabular}

The numbers of nodes added in the final state both with our recovery algorithm and the set cover algorithm are roughly the same. Nonetheless, we can see that our algorithm performs a little bit worse than the set cover algorithm in the less covered area scenarios. Indeed the set cover algorithm takes advantage of its grid layout and perfect spacing between added nodes. However, our homology algorithm gives better result in the more covered scenarios, thanks to the inherent ability of determinantal point processes to locate the coverage holes.

In order to show the advantages of our disaster recovery algorithm we choose to evaluate the compared robustness of the two algorithms when the added nodes positions are slightly moved, i.e. when the practical positioning does not strictly follow the theoretical positioning. In order to do this, we apply a Gaussian perturbation to each the added nodes position. The covariance matrix of the perturbation is given by $\Sigma=\sigma^{2} \mathrm{Id}$ with $\sigma^{2}=0.01$, which means that the standard deviation for each node is of $\sigma=0.1$. Other simulations parameters are unchanged, results in Table VI are given in mean over $10^{4}$ simulations. First, we compute the average number of holes $\mathbf{E}\left[\beta_{1}\right]$ created by the Gaussian perturbation. Then, we count the percentage of simulations in which the number of holes is still zero, $\mathbf{P}\left(\beta_{1}=0\right)$, after the Gaussian perturbation on the new nodes positions.

\begin{tabular}{|ccccc|}
\hline \% of area initially covered & $20 \%$ & $40 \%$ & $60 \%$ & $80 \%$ \\
\hline \hline \multicolumn{5}{|c|}{$\mathbf{E}\left[\beta_{1}\right]$} \\
\hline Set Cover algorithm & 0.68 & 0.67 & 0.48 & 0.35 \\
\hline Homology algorithm & 0.58 & 0.52 & 0.36 & 0.26 \\
\hline \hline \multicolumn{5}{|}{$\mathbf{P}\left(\beta_{1}=0\right)$} \\
\hline Set Cover algorithm & $40.7 \%$ & $45.2 \%$ & $58.8 \%$ & $68.9 \%$ \\
\hline Homology algorithm & $54.0 \%$ & $58.0 \%$ & $68.8 \%$ & $76.1 \%$ \\
\hline
\end{tabular}

TABLE VI

$\mathbf{E}\left[\beta_{1}\right]$ And $\mathbf{P}\left(\beta_{1}=0\right)$ After the Gaussian perturbation

We can see that the perturbation on the number of holes decreases with the percentage of area initially covered, since the initial nodes are not perturbed. Our homology algorithm clearly performs better. Even in the least covered scenarios, there are less than half of the simulations that create coverage holes, which is not the case for the set cover algorithm. The set cover algorithm also always create more coverage holes in mean than our homology algorithm for the same nodes positions perturbation. Therefore our algorithm seems more fitted to the disaster recovery case when a recovery network is deployed in emergency both indoor, via Femtocells, and outdoor, via a trailer fleet, where exact GPS locations are not always available, and exact theoretical positioning is not always followed.

\section{CONCLUSION}

In this paper we propose three simplicial homology-based algorithms that meet three problems of future cellular networks equipped with SON technology. First, we propose a frequency auto-planning algorithm that uses simplicial homology to provide a homogeneous usage of frequencies. Then, we present an energy conservation algorithm that adapt the stay-on nodes of a cellular network to both coverage, computed via simplicial homology, and the capacity defined by user demand. Finally, we introduce a disaster recovery algorithm in which we use simplicial homology to compute coverage, and the repulsive Ginibre determinantal point process to propose an efficient fixing to a damaged network.

We investigate the performance of these three homologybased algorithm. We compute their complexity that is highly dependent on the simplicial complex representation which is necessary to the topology computation. We compare our three homology-based greedy algorithms to three graph-based greedy algorithms to exhibit the benefits of using simplicial homology. We show the advantages of our algorithms, which are not mathematical optimality but application oriented. Indeed, some tradeoffs in complexity and performance are necessary in order to obtain results more suited to the applications encountered in future cellular networks. Despite the fact that the optimal number of frequencies is not always reached, our frequency auto-planning algorithm provides an even and better coverage for every planned frequency than traditionnal methods. Our energy conservation algorithm is the first to take into account both user traffic and coverage. And even if a square grid method is mathematically optimal, our disaster recovery algorithm offers a repaired network robust to slight modifications of its nodes positions and is able to target coverage holes.

As of future work, it is first possible to consider a more complex model for the cellular network where some nodes location are known. Indeed, with the arrival of HetNet, every node locations is not always node, however the operator has still full knowledge of the eNBs locations. Then it would be possible to mix a homological approach to a geometrical approach for the coverage computation, and some gains in complexity could be achieved. Secondly, a next step is to derive more advanced algorithms from our homology-based approach. Gains in both complexity and performance are likely, and comparison with more elaborate graph-based algorithm would be possible. 


\section{REFERENCES}

[1] 3GPP, "TR 36.913: Requirements for further advancements for evolved universal terrestrial radio access (E-UTRA)," Tech. Rep., Mar. 2009.

[2] S. Hmlinen, H. Sanneck, and C. Sartori, LTE Self-Organising Networks (SON): Network Management Automation for Operational Efficiency, 1st ed. Wiley Publishing, 2012.

[3] S. Haykin, "Cognitive radio: brain-empowered wireless communications," Selected Areas in Communications, IEEE Journal on, vol. 23 no. 2, pp. 201-220, 2005.

[4] D. Lopez-Perez, A. Valcarce, G. de la Roche, and J. Zhang, "Ofdma femtocells: A roadmap on interference avoidance," Communications Magazine, IEEE, vol. 47, no. 9, pp. 41-48, September 2009.

[5] A. Vergne, L. Decreusefond, and P. Martins, "Reduction algorithm for simplicial complexes," in INFOCOM, 2013 Proceedings IEEE, 2013, pp. $475-479$.

[6] A. Vergne, I. Flint, L. Decreusefond, and P. Martins, "Disaster Recovery in Wireless Networks: A Homology-Based Algorithm," in ICT 2014, May 2014.

[7] J. Li and R. Jantti, "On the study of self-configuration neighbour cell list for mobile WiMAX," Next Generation Mobile Applications, Services and Technologies, International Conference on, vol. 0, pp. 199-204, 2007.

[8] D. Kim, B. Shin, D. Hong, and J. Lim, "Self-configuration of neighbor cell list utilizing E-UTRAN nodeB scanning in LTE systems," in Consumer Communications and Networking Conference (CCNC), 2010 7th IEEE, 2010, pp. 1-5.

[9] S. Irani, "Coloring inductive graphs on-line," Algorithmica, vol. 11 , no. 1 , pp. 53-72, 1994. [Online]. Available: http://dx.doi.org/10.1007/BF01294263

[10] A. Nasif and B. Mark, "Opportunistic spectrum sharing with multiple cochannel primary transmitters," Wireless Communications, IEEE Transactions on, vol. 8, no. 11, pp. 5702-5710, 2009.

[11] S. Das, S. Sen, and R. Jayaram, "A structured channel borrowing scheme for dynamic load balancing in cellular networks," in Distributed Computing Systems, 1997., Proceedings of the 17th International Conference on, 1997, pp. 116-123.

[12] T. Al-Meshhadany and K. Al-Agha, "VCB by means of soft2hard handover in WCDMA," in Mobile and Wireless Communications Network, 2002. 4th International Workshop on, 2002, pp. 487-491.

[13] T. Fujii and S. Nishioka, "Selective handover for traffic balance in mobile radio communications," in Communications, 1992. ICC '92, Conference record, SUPERCOMM/ICC '92, Discovering a New World of Communications., IEEE International Conference on, 1992, pp. 18401846 vol.4.

[14] L. Du, J. Bigham, and L. Cuthbert, "An intelligent geographic load balance scheme for mobile cellular networks," in Computer Communications and Networks, 2002. Proceedings. Eleventh International Conference on, 2002, pp. 348-353.

[15] S. Das, H. Viswanathan, and G. Rittenhouse, "Dynamic load balancing through coordinated scheduling in packet data systems," in INFOCOM 2003. Twenty-Second Annual Joint Conference of the IEEE Computer and Communications. IEEE Societies, vol. 1, 2003, pp. 786-796 vol.1.

[16] P. Jiang, J. Bigham, and J. Wu, "Self-organizing relay stations in relay based cellular networks," Comput. Commun., vol. 31, no. 13, pp. 2937-2945, Aug. 2008. [Online]. Available: http://dx.doi.org/10.1016/j.comcom.2008.02.024

[17] I. Ashraf, L. T. W. Ho, and H. Claussen, "Improving energy efficiency of femtocell base stations via user activity detection," in Wireless Communications and Networking Conference (WCNC), 2010 IEEE, 2010, pp. 1-5.

[18] E. Campos-Nañez, A. Garcia, and C. Li, "A game-theoretic approach to efficient power management in sensor networks," Oper. Res., vol. 56, no. 3, pp. 552-561, 2008. [Online]. Available: http://dx.doi.org/10.1287/opre.1070.0435

[19] C. Mueller, M. Kaschub, C. Blankenhorn, and S. Wanke, "A cell outage detection algorithm using neighbor cell list reports," in Self-Organizing Systems, ser. Lecture Notes in Computer Science, K. Hummel and J. Sterbenz, Eds. Springer Berlin Heidelberg, 2008, vol. 5343, pp. 218-229. [Online]. Available: http://dx.doi.org/10.1007/978-3-540-92157-8_19

[20] M. Amirijoo, L. Jorguseski, T. Kurner, R. Litjens, M. Neuland, L. Schmelz, and U. Turke, "Cell outage management in LTE networks," in Wireless Communication Systems, 2009. ISWCS 2009. 6th International Symposium on, 2009, pp. 600-604.

[21] 3GPP, "Telecommunications management; self-healing OAM; concepts and requirements," Tech. Rep., vol. 3GPP TS 32.541 v1.6.1, 2010.
[22] K. Morrison, "Rapidly recovering from the catastrophic loss of a major telecommunications office," Communications Magazine, IEEE, vol. 49, no. 1, pp. 28-35, 2011.

[23] V. Chvatal, "A Greedy Heuristic for the Set-Covering Problem," Mathematics of Operations Research, vol. 4, no. 3, pp. 233-235, 1979. [Online]. Available: http://dx.doi.org/10.2307/3689577

[24] D. Haussler and E. Welzl, "Epsilon-nets and simplex range queries," in Proceedings of the second annual symposium on Computational geometry, ser. SCG '86. New York, NY, USA: ACM, 1986, pp. 61-71. [Online]. Available: http://doi.acm.org/10.1145/10515.10522

[25] Q. Fang, J. Gao, L. Guibas, V. de Silva, and L. Zhang, "GLIDER: Gradient landmark-based distributed routing for sensor networks," in Proc. IEEE Conference on Computer Communications (INFOCOM), 2005.

[26] A.Nguyen, N.Milosavljević, Q.Fang, J.Gao, and L.J.Guibas, "Landmark selection and greedy landmark-descent routing for sensor networks," in Proceedings of IEEE INFOCOM 2007, 2007.

[27] O. Aliu, A. Imran, M. Imran, and B. Evans, "A survey of self organisation in future cellular networks," Communications Surveys Tutorials, IEEE, vol. 15, no. 1, pp. 336-361, 2013.

[28] A. Hatcher, Algebraic Topology. Cambridge University Press, 2002.

[29] F. Yan, P. Martins, and L. Decreusefond, "Accuracy of homology based approaches for coverage hole detection in wireless sensor networks," in ICC 2012, Jun. 2012

[30] L. Decreusefond, P. Martins, and A. Vergne, "Reduction algorithm for random abstract simplicial complexes," Mar. 2014, hal-00864303. [Online]. Available: http://hal.archives-ouvertes.fr/hal-00864303

[31] L. Decreusefond, I. Flint, and A. Vergne, "Efficient simulation of the Ginibre process," Oct. 2013, hal-00869259. [Online]. Available: http://hal.archives-ouvertes.fr/hal-00869259

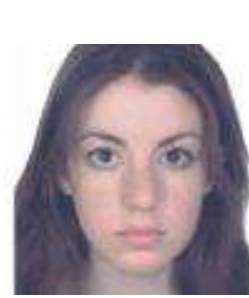

Anaïs Vergne received the Dipl.Ing. degree in telecommunications from Telecom ParisTech, Paris, France in 2010. She obtained the Ph.D. degree in networking and computer sciences in 2013 from Telecom ParisTech, Paris, France. She is currently a post-doctoral fellow in the Geometrica team at Inria Saclay - Ile de France, Palaiseau, France. Her research interests include stochastic geometry applications to wireless networks, more particularly algebraic topology applied to wireless sensor networks.

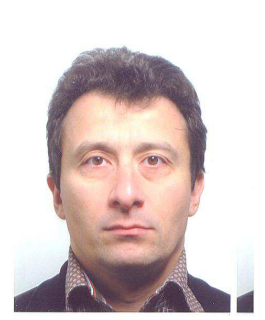

Laurent Decreusefond is a former student of Ecole Normale Supérieure de Cachan. He obtained his Ph.D. degree in Mathematics in 1994 from Telecom ParisTech and his Habilitation in 2001. He is currently a Professor in the Network and Computer Science Department, at Telecom ParisTech. His main fields of interest are the Malliavin calculus, the stochastic analysis of long range dependent processes, random geometry and topology and their applications. With P. Moyal, he co-authored a book about the stochastic modeling of telecommunication.

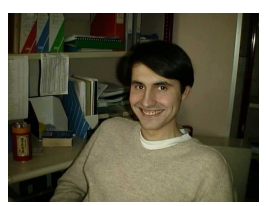

Philippe Martins received a M.S. degree in signal processing and another M.S. degree in networking and computer science from Orsay University and ESIGETEL France, in 1996. He received the Ph.D. degree in electrical engineering from Telecom ParisTech, Paris, France, in 2000. He is currently a Professor in the Network and Computer Science Department, at Telecom Paris- Tech. His main research interests lie in performance evaluation in wireless networks (RRM, scheduling, handover algorithms, radio metrology). His current investigations address mainly three issues: a) the design of distributed sensing algorithms for cognitive radio b) distributed coverage holes detection in wireless sensor networks c) the definition of analytical models for the planning and the dimensioning of cellular systems. He has published several papers on different international journals and conferences. He is also an IEEE senior member and he is co-author of several books on $3 \mathrm{G}$ and $4 \mathrm{G}$ systems. 